Article

\title{
Shannon Type Inequalities for Kapur's Entropy
}

\author{
Bo-Yan Xi ${ }^{1} \oplus$, Dan-Dan Gao ${ }^{1}\left(\right.$, Tao Zhang ${ }^{2} \oplus$, Bai-Ni Guo ${ }^{3, *}$ and Feng $\mathrm{Qi}^{4} \oplus$
}

1 College of Mathematics, Inner Mongolia University for Nationalities, Tongliao 028043, China; baoyintu78@imun.edu.cn (B.-Y.Xi); Dan-DanGao@hotmail.com (D.-D.Gao)

2 School of Mathematical Sciences, Inner Mongolia University, Hohhot 010021, China; zhangtaocx@163.com

3 School of Mathematics and Informatics, Henan Polytechnic University, Jiaozuo 454010, China

4 School of Mathematical Sciences, Tianjin Polytechnic University, Tianjin 300387, China; qifeng618@gmail.com

* Correspondence: bai.ni.guo@gmail.com

Received: 25 November 2018; Accepted: 24 December 2018; Published: 26 December 2018 updates

\begin{abstract}
In the paper, by methods of the theory of majorization, the authors establish the Schur $m$-convexity and Shannon type inequalities for Kapur's entropy.
\end{abstract}

Keywords: Kapur's entropy; Shannon type inequality; Schur m-convexity; majorization

MSC: 26A51; 26D15; 26E60; 94A17

\section{Introduction and Main Results}

Let $p=\left(p_{1}, p_{2}, \ldots, p_{n}\right)$ be a probability vector, that is, $p_{i} \geq 0$ for $1 \leq i \leq n$ and $\sum_{i=1}^{n} p_{i}=1$. The quantity

$$
H(p)=-\sum_{i=1}^{n} p_{i} \ln p_{i}
$$

where, if $p_{i}=0$, we put $p_{i} \ln p_{i}=0$ in the sum, is known as entropy of $p$, or the Shannon information entropy of $p$.

Theorem 1 ([1] (p. 101)). Let $p=\left(p_{1}, p_{2}, \ldots, p_{n}\right)$ be a probability vector. Then $H(p)$ is Schur-concave on $[0,1]^{n}$. In particular, we have

$$
H(1,0, \ldots, 0) \leq H(p) \leq H\left(p_{0}\right)=\ln n,
$$

where $p_{0}=\left(\frac{1}{n}, \frac{1}{n}, \ldots, \frac{1}{n}\right)$.

Let $p$ be a probability vector and $t \in(0, \infty)$. The quantity

$$
H_{t}(p)=H_{t}\left(p_{1}, p_{2}, \ldots, p_{n}\right)=-\frac{1}{\sum_{i=1}^{n} p_{i}^{t}} \sum_{i=1}^{n} p_{i}^{t} \ln p_{i}^{t}
$$

is known [2] as Kapur's entropy of order 1 and type $t \in(0, \infty)$. It is easy to see that

$$
H_{t}\left(p_{0}\right)=-\ln A_{n}\left(p_{0}^{t}\right)=t \ln n,
$$

where $p^{t}=\left(p_{1}^{t}, p_{2}^{t}, \ldots, p_{n}^{t}\right)$ and $A_{n}\left(p^{t}\right)=\frac{1}{n} \sum_{i=1}^{n} p_{i}^{t}$. It is easy to see that the inequality $H_{1}(p) \leq \ln n$ holds for each probability vector $p$. However, the inequality

$$
H_{t}(p) \leq \ln n
$$


does not hold for each $t \in(0, \infty)$ and each probability vector $p$. Stolarsky [3] shows that the inequality (1) holds for $t \geq t_{0}(n)$, where $t_{0}(2)=\frac{1}{2}$. Hereafter, Clausing [4] proved that if $n>3$ and $t=t_{0}(n)$, then the inequality (1) holds for each probability vector $p$. Thus, with respect to $t \in(0, \infty)$, the function $H_{t}(p)$ is not strictly Schur-convex. For more information about this topic, the reader is referred to the papers [2,4-14] and the closely related references therein.

In this paper, we will establish the Schur $m$-convexity and some Shannon type inequalities for Kapur's entropy $H_{t}(p)$.

Our main results are the following two theorems.

Theorem 2. Let $p \in(0, \infty)^{n}, t \in(0, \infty)$, and $\lambda_{1}, \lambda_{2}, \ldots, \lambda_{n} \in[0,1]$ with $\sum_{i=1}^{n} \lambda_{i}=1$ for $n \geq 2$. Then the function $H_{t}(p)$ is Schur $m$-concave on $(0, \infty)^{n}$ for $m=t$ and

$$
H_{t}(p) \leq H_{t}\left(M_{t}(1, \lambda ; p), M_{t}(2, \lambda ; p), \ldots, M_{t}(n, \lambda ; p)\right) \leq-\ln A_{n}\left(p^{t}\right)
$$

with the equality in (2) holding for $p_{0}=\left(\frac{1}{n}, \frac{1}{n}, \ldots, \frac{1}{n}\right)$, where

$$
M_{t}(k, \lambda ; p)=\left(\sum_{i=1}^{n} \lambda_{i} p_{i+k-1}^{t}\right)^{1 / t}, \quad k=1,2, \ldots, n
$$

with $p_{n+i}=p_{i}$ for $i=1,2, \ldots, n-1$.

Theorem 3. Let $p \in(0,1)^{n}, t \in(0,1]$, and $\lambda_{1}, \lambda_{2}, \ldots, \lambda_{n} \in[0,1]$ with $\sum_{i=1}^{n} p_{i}=\sum_{i=1}^{n} \lambda_{i}=1$ for $n \geq 1$.

1. If $0<t<\frac{1}{2}$ and $p \in[q, 1)$ with $\frac{1}{q} \geq n$, then

$$
\ln n \leq-\sum_{k=1}^{n}\left[M_{1}(k, \lambda ; p)\right]^{t} \ln M_{1}(k, \lambda ; p)
$$

2. If $0<t<\frac{1}{2}$ and $p \in(0, q]^{n}$ with $\frac{1}{q} \leq n$, or if $\frac{1}{2} \leq t \leq 1$ and $p \in(0,1]^{n}$, then

$$
\frac{H_{t}(p)}{t} \leq-\frac{1}{n} \sum_{k=1}^{n}\left[M_{1}(k, \lambda ; p)\right]^{t} \ln M_{1}(k, \lambda ; p) \leq \min \left\{\frac{1}{t}, n^{1-t}\right\} \ln n,
$$

where $q=e^{(2 t-1) / t(1-t)}$ for $0<t<\frac{1}{2}$ and $M_{1}(k, \lambda ; p)$ is defined by (3).

\section{Definitions and Lemmas}

For proving our main results, we need several definitions and lemmas below.

It is well known that a function $\varphi\left(x_{1}, x_{2}, \ldots, x_{n}\right)$ of $n$ variables is said to be symmetric if its value is unchanged for any permutation of its $n$ variables $x_{1}, x_{2}, \ldots, x_{n}$.

Definition 1 ([1,15]). Let $x=\left(x_{1}, x_{2}, \ldots, x_{n}\right)$ and $y=\left(y_{1}, y_{2}, \ldots, y_{n}\right) \in \mathbb{R}^{n}$.

1. A tuple $x$ is said to be majorized by $y$ (in symbols $x \prec y$ ) if $\sum_{i=1}^{k} x_{[i]} \leq \sum_{i=1}^{k} y_{[i]}$ for $k=1,2, \ldots, n-1$ and $\sum_{i=1}^{n} x_{i}=\sum_{i=1}^{n} y_{i}$, where $x_{[1]} \geq x_{[2]} \geq \cdots \geq x_{[n]}$ and $y_{[1]} \geq y_{[2]} \geq \cdots \geq y_{[n]}$ are rearrangements of $x$ and $y$ in a descending order.

2. A set $\Omega \subseteq \mathbb{R}^{n}$ is called convex if $\left(\lambda x_{1}+(1-\lambda) y_{1}, \lambda x_{2}+(1-\lambda) y_{2}, \ldots, \lambda x_{n}+(1-\lambda) y_{n}\right) \in \Omega$ for each $x, y \in \Omega$ and $\lambda \in[0,1]$.

3. Let $\Omega \subseteq \mathbb{R}^{n}$. A function $\varphi: \Omega \rightarrow \mathbb{R}$ is said to be Schur-convex on $\Omega$ if $x \prec y$ on $\Omega$ implies $\varphi(x) \leq$ $\varphi(y)$. A function $\varphi$ is said to be Schur-concave on $\Omega$ if and only if $-\varphi$ is Schur-convex.

Definition 2 ([16]). Let $\Omega \subseteq(0, \infty)^{n}$ and $\varphi: \Omega \rightarrow(0, \infty)$. 
1. The set $\Omega$ is said to be geometrically convex if $\left(x_{1}^{\lambda} y_{1}^{1-\lambda}, x_{2}^{\lambda} y_{2}^{1-\lambda}, \ldots, x_{n}^{\lambda} y_{n}^{1-\lambda}\right) \in \Omega$ for each $x, y \in \Omega$ and $\lambda \in[0,1]$.

2. The function $\varphi$ is said to be Schur-geometrically convex on $\Omega$ if the majorization $\ln x=\left(\ln x_{1}, \ln x_{2}, \ldots, \ln x_{n}\right) \prec \ln y=\left(\ln y_{1}, \ln y_{2}, \ldots, \ln y_{n}\right)$ implies $\varphi(x) \leq \varphi(y)$ for each $x, y \in \Omega$.

Definition 3 ([17]). Let $\Omega \subseteq(0, \infty)^{n}$ and $\varphi: \Omega \rightarrow(0, \infty)$.

1. The set $\Omega$ is said to be harmonically convex if $\frac{x y}{\lambda x+(1-\lambda) y} \in \Omega$ for each $x, y \in \Omega$ and $\lambda \in[0,1]$.

2. The function $\varphi$ is said to be Schur-harmonically convex on $\Omega$ if the majorization $\frac{1}{x}=\left(\frac{1}{x_{1}}, \frac{1}{x_{2}}, \ldots, \frac{1}{x_{n}}\right) \prec$ $\frac{1}{y}=\left(\frac{1}{y_{1}}, \frac{1}{y_{2}}, \ldots, \frac{1}{y_{n}}\right)$ implies $\varphi(x) \leq \varphi(y)$ for each $x, y \in \Omega$.

Definition 4 ([18-20]). Let $f_{m}(x)=\left\{\begin{array}{ll}\frac{x^{m}-1}{m}, & m \neq 0 ; \\ \ln x, & m=0 .\end{array}\right.$ A function $\varphi: \Omega \subseteq(0, \infty)^{n} \rightarrow \mathbb{R}$ is said to be Schur m-power convex on $\Omega$ if the majorization relation $\left(f_{m}\left(x_{1}\right), f_{m}\left(x_{2}\right), \ldots f_{m}\left(x_{n}\right)\right) \prec$ $\left(f_{m}\left(y_{1}\right), f_{m}\left(y_{2}\right), \ldots f_{m}\left(y_{n}\right)\right)$ on $\Omega$ implies that $\varphi(x) \leq \varphi(y)$.

Remark 1. It is not difficult to see that, when $m \neq 0$,

$$
\left(\frac{x_{1}^{m}-1}{m}, \frac{x_{2}^{m}-1}{m}, \ldots, \frac{x_{n}^{m}-1}{m}\right) \prec\left(x_{1}^{m}-1, x_{2}^{m}-1, \ldots, x_{n}^{m}-1\right) \prec\left(x_{1}^{m}, x_{2}^{m}, \ldots, x_{n}^{m}\right) .
$$

When taking $m=1,0,-1$, we derive that $f_{1}(x)=x-1, f_{0}(x)=\ln x$, and $f_{-1}(x)=1-\frac{1}{x}$ in Definition 4 . Therefore, we can derive from Definition 4 the Schur-convexity, Schur-geometric convexity, and Schur-harmonic convexity, respectively.

Lemma 1 ([1,15,21]). Let $\Omega \subseteq \mathbb{R}^{n}$ be a symmetric convex set with nonempty interior and let $\varphi: \Omega \rightarrow(0, \infty)$ be a continuous symmetric function which is differentiable in the interior $\Omega^{\circ}$. Then $\varphi$ is a Schur-convex function on $\Omega$ if and only if

$$
\left(x_{1}-x_{2}\right)\left[\frac{\partial \varphi(x)}{\partial x_{1}}-\frac{\partial \varphi(x)}{\partial x_{2}}\right] \geq 0, \quad x \in \Omega^{\circ} .
$$

Lemma 2 ([18-20]). Let $\Omega \subseteq(0, \infty)^{n}$ be a symmetric set with nonempty interior $\Omega^{\circ}$ and let $\varphi: \Omega \rightarrow \mathbb{R}$ be continuous on $\Omega$ and differentiable in $\Omega^{\circ}$. Then $\varphi$ is Schur m-power convex on $\Omega$ if and only if $\varphi$ is symmetric on $\Omega$ such that

$$
\frac{x_{1}^{m}-x_{2}^{m}}{m}\left[x_{1}^{1-m} \frac{\partial \varphi(x)}{\partial x_{1}}-x_{2}^{1-m} \frac{\partial \varphi(x)}{\partial x_{2}}\right] \geq 0, \quad m \neq 0
$$

and

$$
\left(\ln x_{1}-\ln x_{2}\right)\left[x_{1} \frac{\partial \varphi(x)}{\partial x_{1}}-x_{2} \frac{\partial \varphi(x)}{\partial x_{2}}\right] \geq 0, \quad m=0
$$

for $x \in \Omega^{\circ}$.

For more information about various convexity named after Schur, please refer to [22-34] and closely related references therein.

\section{Shannon Type Inequalities for Kapur's Entropy}

Now we are in a position to prove our main results.

Proof of Theorem 2. Calculating the partial derivatives of function $H_{t}(p)$ in $p_{1}$ and $p_{2}$ yields

$$
\frac{\partial H_{t}(p)}{\partial p_{1}}=\frac{-t p_{1}^{t-1}}{\left[p_{1}^{t}+p_{2}^{t}+A(p)\right]^{2}}\left[p_{1}^{t}+p_{2}^{t}+A(p)-B(p)+\left(p_{2}^{t}+A(p)\right) \ln p_{1}^{t}-p_{2}^{t} \ln p_{2}^{t}\right]
$$


and

$$
\frac{\partial H_{t}(p)}{\partial p_{2}}=\frac{-t p_{2}^{t-1}}{\left[p_{1}^{t}+p_{2}^{t}+A(p)\right]^{2}}\left[p_{1}^{t}+p_{2}^{t}+A(p)-B(p)+\left(p_{1}^{t}+A(p)\right) \ln p_{2}^{t}-p_{1}^{t} \ln p_{1}^{t}\right]
$$

where $A(p)=\sum_{i=3}^{n} p_{i}^{t}$ and $B(p)=\sum_{i=3}^{n} p_{i}^{t} \ln p_{i}^{t}$ under the condition that any empty sum is understood as null.

Letting $t=m$ in Definition 4 and utilizing Lemma 2 give

$$
\begin{aligned}
& \frac{p_{1}^{m}-p_{2}^{m}}{m}\left[p_{1}^{1-m} \frac{\partial H_{t}(p)}{\partial p_{1}}-p_{2}^{1-m} \frac{\partial H_{t}(p)}{\partial p_{2}}\right] \\
= & -\frac{t\left(p_{1}^{m}-p_{2}^{m}\right)}{m\left(p_{1}^{t}+p_{2}^{t}+A(p)\right)^{2}}\left[\left(p_{1}^{t}+p_{2}^{t}+A(p)-B(p)\right)\left(p_{1}^{t-m}-p_{2}^{t-m}\right)\right. \\
& \left.+A(p)\left(p_{1}^{t-m} \ln p_{1}^{t}-p_{2}^{t-m} \ln p_{2}^{t}\right)+p_{1}^{t-m} p_{2}^{t-m}\left(p_{1}^{m}+p_{2}^{m}\right)\left(\ln p_{1}^{t}-\ln p_{2}^{t}\right)\right] \\
= & -\frac{p_{1}^{t}-p_{2}^{t}}{\left(p_{1}^{t}+p_{2}^{t}+A(p)\right)^{2}}\left[A(p)\left(\ln p_{1}^{t}-\ln p_{2}^{t}\right)+\left(p_{1}^{t}+p_{2}^{t}\right)\left(\ln p_{1}^{t}-\ln p_{2}^{t}\right)\right] \\
= & -\frac{1}{\left(p_{1}^{t}+p_{2}^{t}+A(p)\right)^{2}}\left(p_{1}^{t}-p_{2}^{t}\right)\left(\ln p_{1}^{t}-\ln p_{2}^{t}\right) \sum_{i=1}^{n} p_{i}^{t} \\
\leq & 0 .
\end{aligned}
$$

Hence, the function $H_{t}(p)$ is Schur $m$-concave on $(0, \infty)^{n}$ for $m=t$.

For $\ell_{1}, \ell_{2}, \ldots, \ell_{k} \in\{1,2, \ldots, n\}$ and $1 \leq k \leq n$, we have

$$
\sum_{j=1}^{k} p_{[j]}^{t}-\sum_{j=1}^{k} \sum_{i=1}^{n} \lambda_{i} p_{i+\ell_{j}-1}^{t}=\sum_{j=1}^{k} p_{[j]}^{t}-\sum_{i=1}^{n} \lambda_{i} \sum_{j=1}^{k} p_{i+\ell_{j}-1}^{t} \geq \sum_{j=1}^{k} p_{[j]}^{t}-\sum_{i=1}^{n} \lambda_{i} \sum_{j=1}^{k} p_{[j]}^{t}=0 .
$$

Let $y_{j}=\sum_{i=1}^{n} \lambda_{i} p_{i+j-1}^{t}$ for $j=1,2, \ldots, n$. If there exists $1 \leq k_{0}<n$ such that $\sum_{j=1}^{k_{0}} y_{[j]} \geq k_{0} A_{n}\left(p^{t}\right)$ and $\sum_{j=1}^{k_{0}+1} y_{[j]}<\left(k_{0}+1\right) A_{n}\left(p^{t}\right)$, that is,

$$
\sum_{i=1}^{n} p_{i}^{t}=\sum_{j=1}^{k_{0}+1} y_{[j]}+\sum_{k_{0}+2}^{n} y_{[j]}<\left(k_{0}+1\right) A_{n}\left(p^{t}\right)+\left(n-k_{0}-1\right) A_{n}\left(p^{t}\right)=\sum_{i=1}^{n} p_{i}^{t}
$$

Then, it follows that

$$
\left(A_{n}\left(p^{t}\right), A_{n}\left(p^{t}\right), \ldots, A_{n}\left(p^{t}\right)\right) \prec\left(\left[M_{t}(1, \lambda ; p)\right]^{t},\left[M_{t}(2, \lambda ; p)\right]^{t}, \ldots,\left[M_{t}(n, \lambda ; p)\right]^{t}\right) \prec\left(p_{1}^{t}, p_{2}^{t}, \ldots, p_{n}^{t}\right) .
$$

Further by virtue of Definition 4, we have

$$
H_{t}(p) \leq H_{t}\left(M_{t}(1, \lambda ; p), M_{t}(2, \lambda ; p), \ldots, M_{t}(n, \lambda ; p)\right) \leq-\ln A_{n}\left(p^{t}\right) .
$$

The proof of Theorem 2 is thus complete.

Proof of Theorem 3. For all $p \in(0,1)^{n}$ and $t \in(0,1]$ with $\sum_{i=1}^{n} p_{i}=1$, by the easily-understandable inequality

$$
A_{n}\left(p^{t}\right) \geq \frac{1}{n} \sum_{i=1}^{n} p_{i}=\frac{1}{n}
$$

for $0<t \leq 1$, we obtain

$$
-\frac{1}{n^{1-t}} \sum_{i=1}^{n} p_{i}^{t} \ln p_{i}^{t} \leq H_{t}(p) \leq-\sum_{i=1}^{n} p_{i}^{t} \ln p_{i}^{t}
$$


Let $F_{t}(p)=-\sum_{i=1}^{n} p_{i}^{t} \ln p_{i}^{t}$. Then

$$
\frac{\partial F_{t}(p)}{\partial p_{1}}=-t p_{1}^{t-1}\left(1+\ln p_{1}^{t}\right) \quad \text { and } \quad \frac{\partial F_{t}(p)}{\partial p_{2}}=-t p_{2}^{t-1}\left(1+\ln p_{2}^{t}\right) .
$$

By Lemma 1, we obtain

$$
\left(p_{1}-p_{2}\right)\left[\frac{\partial F_{t}(p)}{\partial p_{1}}-\frac{\partial F_{t}(p)}{\partial p_{2}}\right]=t\left(p_{1}-p_{2}\right)\left[g\left(p_{2}\right)-g\left(p_{1}\right)\right]
$$

where $g(p)=p^{t-1}+p^{t-1} \ln p^{t}$ for $p \in(0,1]$. Then

1. when $0<t<\frac{1}{2}$, the function $g(p)$ is increasing on $(0, q]$ and decreasing on $[q, 1)$;

2. when $\frac{1}{2} \leq t \leq 1$, the function $g(p)$ is increasing on $(0,1]$.

Therefore, by Lemma 1, we find that

1. if $0<t<\frac{1}{2}$, the function $F_{t}(p)$ is Schur-convex on $[q, 1)^{n}$;

2. if $0<t<\frac{1}{2}$, the function $F_{t}(p)$ is Schur-concave on $(0, q]^{n}$;

3. if $\frac{1}{2} \leq t \leq 1$, the function $F_{t}(p)$ is Schur-concave on $(0,1]^{n}$.

For $\lambda_{1}, \lambda_{2}, \ldots, \lambda_{n} \in[0,1]$ with $\sum_{i=1}^{n} \lambda_{i}=1$, we have

$$
\left(\frac{1}{n}, \frac{1}{n}, \ldots, \frac{1}{n}\right) \prec\left(\sum_{i=1}^{n} \lambda_{i} p_{i}, \sum_{i=1}^{n} \lambda_{i} p_{i+1}, \ldots, \sum_{i=1}^{n} \lambda_{i} p_{i+n-1}\right) \prec p .
$$

By Definition 1, we see that

1. if $0<t<\frac{1}{2}$ and $p \in[q, 1)^{n}$ with $\frac{1}{q} \geq n$, then

$$
H_{t}(p) \geq n^{t-1} F_{t}(p) \geq-\frac{1}{n^{1-t}} \sum_{k=1}^{n}\left[M_{1}(k, \lambda ; p)\right]^{t} \ln \left[M_{1}(k, \lambda ; p)\right]^{t} \geq \frac{t \ln n}{n} ;
$$

2. if $0<t<\frac{1}{2}$ and $p \in(0, q]^{n}$ with $\frac{1}{q} \leq n$, then

$$
H_{t}(p) \leq F_{t}(p) \leq-\sum_{k=1}^{n}\left[M_{1}(k, \lambda ; p)\right]^{t} \ln \left[M_{1}(k, \lambda ; p)\right]^{t} \leq t n^{1-t} \ln n
$$

3. if $\frac{1}{2} \leq t \leq 1$ and $p \in(0,1)^{n}$, the inequality (5) still holds.

The proof of Theorem 3 is thus complete.

\section{Remarks}

Finally we list several remarks on our main results and closely related ones.

Remark 2. Let $p \in(0, \infty)^{n}, t \geq 1, \lambda_{1}, \lambda_{2}, \ldots, \lambda_{n} \in[0,1]$ with $\sum_{i=1}^{n} \lambda_{i}=1$ for $n \geq 2$. Since $\left[A_{n}(p)\right]^{t} \leq$ $A_{n}\left(p^{t}\right)$ for $t \geq 1$, from Theorem 2, it follows that

$$
H_{t}(p) \leq H_{t}\left(M_{t}(1, \lambda ; p), M_{t}(2, \lambda ; p), \ldots, M_{t}(n, \lambda ; p)\right) \leq-\ln A_{n}\left(p^{t}\right) \leq-t \ln A_{n}(p)
$$

with equality for $p_{1}=p_{2}=\cdots=p_{n}>0$, where $M_{1}(k, \lambda ; p)$ is defined by (3).

Remark 3. Let $p \in(0,1)^{n}, t \in(0, \infty)$, and $\lambda_{1}, \lambda_{2}, \ldots, \lambda_{n} \in[0,1]$ with $\sum_{i=1}^{n} p_{i}=\sum_{i=1}^{n} \lambda_{i}=1$ and $n \geq 2$. From Remark 2 and the inequality (4), we conclude the following results. 
1. If $t \geq 1$, we have

$$
H_{t}(p) \leq H_{t}\left(M_{t}(1, \lambda ; p), M_{t}(2, \lambda ; p), \ldots, M_{t}(n, \lambda ; p)\right) \leq-\ln A_{n}\left(p^{t}\right) \leq t \ln n
$$

with equality for $p_{0}=\left(\frac{1}{n}, \frac{1}{n}, \ldots, \frac{1}{n}\right)$.

2. If $0<t<1$, we have

$$
H_{t}(p) \leq H_{t}\left(M_{t}(1, \lambda ; p), M_{t}(2, \lambda ; p), \ldots, M_{t}(n, \lambda ; p)\right) \leq-\ln A_{n}\left(p^{t}\right) \leq \ln n .
$$

In particular, when $\lambda_{1}=\lambda_{2}=\frac{1}{2}$ and $\lambda_{3}=\cdots=\lambda_{n}=0$,

1. if $t \geq 1$, then

$$
H_{t}(p) \leq H_{t}\left(\sqrt[t]{\frac{p_{1}^{t}+p_{2}^{t}}{2}}, \sqrt[t]{\frac{p_{2}^{t}+p_{3}^{t}}{2}}, \ldots, \sqrt[t]{\frac{p_{n}^{t}+p_{1}^{t}}{2}}\right) \leq-\ln A_{n}\left(p^{t}\right) \leq t \ln n
$$

with equality for $p_{0}=\left(\frac{1}{n}, \frac{1}{n}, \ldots, \frac{1}{n}\right)$;

2. if $0<t<1$, then

$$
H_{t}(p) \leq H_{t}\left(\sqrt[t]{\frac{p_{1}^{t}+p_{2}^{t}}{2}}, \sqrt[t]{\frac{p_{2}^{t}+p_{3}^{t}}{2}}, \ldots, \sqrt[t]{\frac{p_{n}^{t}+p_{1}^{t}}{2}}\right) \leq-\ln A_{n}\left(p^{t}\right) \leq \ln n .
$$

Remark 4. When $0<t<1$, the inequality (6) does not necessarily hold. For $t=0.369, \lambda_{1}=1, \lambda_{2}=\lambda_{3}=0$, and $p=(0.3,0.69,0.01)$, we have

$$
0.4053 \ldots=t \ln n<H_{t}(p)=0.4215 \ldots<\ln n=1.0986 \ldots
$$

Remark 5. Under conditions of Theorems 2 and 3 , if $0<t<\frac{1}{2}$ and $p \in[q, 1)$ with $\frac{1}{q} \geq n$, then

$$
\begin{aligned}
\frac{t \ln n}{n} \leq-\frac{1}{n} \sum_{k=1}^{n}\left[M_{1}(k, \lambda ; p)\right]^{t} \ln \left[M_{1}(k, \lambda ; p)\right]^{t} & \leq H_{t}(p) \\
& \leq H_{t}\left(M_{t}(1, \lambda ; p), M_{t}(2, \lambda ; p), \ldots, M_{t}(n, \lambda ; p)\right) \leq-\ln A_{n}\left(p^{t}\right) \leq \min \left\{1, t n^{1-t}\right\} \ln n .
\end{aligned}
$$

Author Contributions: The authors contributed equally to this work. All authors read and approved the final manuscript.

Funding: This research was supported by National Natural Science Foundation of China (Grant No. 11361038), by Foundation of Research Program of Science and Technology at Universities of Inner Mongolia Autonomous Region (Grant No. NJZZ18154), and by Natural Science Foundation of Inner Mongolia (Grant No. 2018LH01002) in China.

Acknowledgments: The authors are thankful to the anonymous referees for their careful corrections to and valuable comments on the original version of this paper.

Conflicts of Interest: The authors declare no conflict of interest.

\section{References}

1. Marshall, A.W.; Olkin, I.; Arnold, B.C. Inequalities: Theory of Majorization and Its Applications, 2nd ed.; Springer Verlag: New York, NY, USA; Dordrecht, The Netherlands; Heidelberg, Germany; London, UK, 2011; doi:10.1007/978-0-387-68276-1.

2. Kapur, J.N. On some properties of generalised entropies. Indian J. Math. 1967, 9, 427-442.

3. Stolarsky, K.B. A stronger logarithmic inequality suggested by the entropy inequality. SIAM J. Math. Anal. 1980, 11, 242-247. [CrossRef] 
4. Clausing, A. Type $t$ entropy and majorization. SIAM J. Math. Anal. 1983, 14, 203-208. [CrossRef]

5. Bebiano, N.; Lemos, R.; da Providência, J. Inequalities for quantum relative entropy. Linear Algebra Its Appl. 2005, 401, 159-172. [CrossRef]

6. Blachman, N.M. The convolution inequality for entropy powers. IEEE Trans. Inf. Theory 1965, IT-11, $267-271$. [CrossRef]

7. Bobkov, S.G.; Chistyakov, G.P. Entropy power inequality for the Rényi entropy. IEEE Trans. Inf. Theory 2015, 61, 708-714. [CrossRef]

8. Capocelli, R.M.; Taneja, I.J. On some inequalities and generalized entropies: A unified approach. Cybern. Syst. 1985, 16, 341-376. [CrossRef]

9. Cohen, J.E.; Derriennic, Y.; Zbăganu, G. Majorization, monotonicity of relative entropy, and stochastic matrices, Doeblin and Modern Probability (Blaubeuren, 1991). Contemp. Math. 1993, 149, 251-259. [CrossRef]

10. Furuichi, S.; Minculete, N.; Mitroi, F.-C. Some inequalities on generalized entropies. J. Inequal. Appl. 2012, 2012, 226. [CrossRef]

11. Kapur, J.N. Some properties of entropy of order $\alpha$ and type $\beta$. Proc. Indian Acad. Sci. Sect. A 1969, 69, $201-211$. [CrossRef]

12. Mokshay, M.; Andrew, B. Generalized entropy power inequalities and monotonicity properties of information. IEEE Trans. Inf. Theory 2007, 53, 2317-2329. [CrossRef]

13. Qi, F.; Lim, D.; Guo, B.-N. Explicit formulas and identities for the Bell polynomials and a sequence of polynomials applied to differential equations. Rev. Real Acad. Cienc. Exactas Fís. Nat. Ser. A Mat. 2019, in press. [CrossRef]

14. Yao, Y.; Yao, J.-C.; Liou, Y.-C.; Postolache, M. Iterative algorithms for split common fixed points of demicontractive operators without priori knowledge of operator norms. Carpathian J. Math. 2018, 34, 459-466.

15. Wang, B.Y. Foundations of Majorization Inequalities; Beijing Normal Univ. Press: Beijing, China, 1990. (In Chinese)

16. Chu, Y.-M.; Zhang, X.-M.; Wang, G.-D. The Schur geometrical convexity of the extended mean values. J. Convex Anal. 2008, 15, 707-718.

17. Xia, W.F.; Chu, Y.M. Schur-convexity for a class of symmetric functions and its applications. J. Inequal. Appl. 2009, 2009, 493759. [CrossRef]

18. Yang, Z.-H. Schur power convexity of Gini means. Bull. Korean Math. Soc. 2013, 50, 485-498. [CrossRef]

19. Yang, Z.-H. Schur power convexity of Stolarsky means. Publ. Math. Debr. 2012, 80, 43-66. [CrossRef]

20. Yang, Z.-H. Schur power convexity of the Daróczy means. Math. Inequal. Appl. 2013, 16, 751-762. [CrossRef]

21. Niculescu, C.P.; Persson, L.-E. Convex Functions and Their Applications: A Contemporary Approach, 2nd ed.; CMS Books in Mathematics/Ouvrages de Mathématiques de la SMC; Springer: Cham, Switzerland, 2018; doi:10.1007/978-3-319-78337-6.

22. Qi, F. A note on Schur-convexity of extended mean values. Rocky Mt. J. Math. 2005, 35, 1787-1793. [CrossRef]

23. Qi, F.; Niu, D.-W.; Guo, B.-N. Some identities for a sequence of unnamed polynomials connected with the Bell polynomials. Rev. Real Acad. Cienc. Exactas Fís. Nat. Ser. A Mat. 2019, in press. [CrossRef]

24. Qi, F.; Sándor, J.; Dragomir, S.S.; Sofo, A. Notes on the Schur-convexity of the extended mean values. Taiwan. J. Math. 2005, 9, 411-420. [CrossRef]

25. Qi, F.; Shi, X.-T.; Mahmoud, M.; Liu, F.-F. Schur-convexity of the Catalan-Qi function related to the Catalan numbers. Tbil. Math. J. 2016, 9, 141-150. [CrossRef]

26. Rovenţa, I. Schur convexity of a class of symmetric functions. Ann. Univ. Craiova Ser. Mat. Inform. 2010, 37, $12-18$.

27. Shi, H.-N.; Jiang, Y.-M.; Jiang, W.-D. Schur-convexity and Schur-geometrically concavity of Gini mean. Comput. Math. Appl. 2009, 57, 266-274. [CrossRef]

28. Shi, H.-N.; Wu, S.-H.; Qi, F. An alternative note on the Schur-convexity of the extended mean values. Math. Inequal. Appl. 2006, 9, 219-224. [CrossRef]

29. Shi, H.-N.; Zhang, J. Compositions involving Schur harmonically convex functions. J. Comput. Anal. Appl. 2017, 22, 907-922.

30. Sun, J.; Sun, Z.-L.; Xi, B.-Y.; Qi, F. Schur-geometric and Schur-harmonic convexity of an integral mean for convex functions. Turk. J. Anal. Number Theory 2015, 3, 87-89. [CrossRef]

31. Wu, Y.; Qi, F. Schur-harmonic convexity for differences of some means. Analysis 2012, 32, 263-270. [CrossRef] 
32. Wu, Y.; Qi, F.; Shi, H.-N. Schur-harmonic convexity for differences of some special means in two variables. J. Math. Inequal. 2014, 8, 321-330. [CrossRef]

33. Yao, Y.; Qin, X.; Yao, J.-C. Projection methods for firmly type nonexpansive operators. J. Nonlinear Convex Anal. 2018, 19, 407-415.

34. Zhang, X.-M. Geometrically Convex Functions; An'hui University Press: Hefei, China, 2004. (In Chinese)

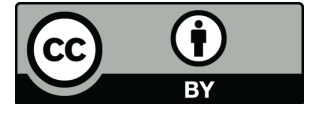

(C) 2018 by the authors. Licensee MDPI, Basel, Switzerland. This article is an open access article distributed under the terms and conditions of the Creative Commons Attribution (CC BY) license (http:/ / creativecommons.org/licenses/by/4.0/). 